\title{
Chiral imbalance in QCD
}

\author{
Alexander Andrianov ${ }^{1,2, \star}$, Vladimir Andrianov ${ }^{1, \star \star}$, and Domenec Espriu ${ }^{2, \star \star \star}$ \\ ${ }^{1}$ Department of Physics, Saint Petersburg State University, \\ Universitetskaya nab. 7/9, Saint Petersburg 199034, Russia, \\ ${ }^{2}$ Institute of Cosmos Sciences (ICCUB), University of Barcelona, \\ Martí i Franquès, 1, 08028 Barcelona, Spain
}

\begin{abstract}
The chiral imbalance (ChI) is given by a difference between the numbers of RH and LH quarks which may occur in the fireball after heavy ion collision. To characterize it adiabatically a quark chiral (axial) chemical potential must be introduced taking into account emergence of a $\mathrm{ChI}$ in such a phase. In this report the phenomenology of formation of Local spatial Parity Breaking (LPB) in the hot and dense baryon matter is discussed and its simulation within a number of QCD-inspired models is outlined. The appearance of new states in the spectra of scalar, pseudoscalar and vector particles in such a matter is elucidated. In particular, from the effective vector meson theory in the presence of Chern-Simons interaction it is demonstrated that the spectrum of massive vector mesons splits into three polarization components with different effective masses. The asymmetry in production of longitudinally and transversely polarized states of $\rho$ and $\omega$ mesons for various values of the dilepton invariant mass can serve as a characteristic indication of the LPB in PHENIX, STAR and ALICE experiments.
\end{abstract}

\section{Introduction: Chiral Imbalance in Heavy lons Collisions}

The behaviour of baryonic matter under extreme conditions in HIC has received a lot of attention $[1,2]$. New properties of QCD in the environment were tested in current accelerator experiments on RHIC and LHC [3]. A medium generated in these collisions (a fireball) may serve for experimental and theoretical studies of various phases of a matter.

The dedicated experimental study of hadron correlations in non-central heavy-ion collisions at RHIC [4] and LHC [5] revealed a signal of the separation of electric charges predicted in [6] as a signature of local P- and CP-odd fluctuations in QCD matter. The subsequent studies [7, 8] improved the theoretical understanding of the underlying phenomenon, the "chiral magnetic effect" (CME) in the reactions for peripheral ion collisions (see [9] for a review).

On the contrary, a gradient density of isosinglet pseudoscalar condensate can be formed as a result of large, "long-lived" topological fluctuations of gluon fields in the fireball in central collisions (see [10] for details). To describe various effects of hadron matter in a fireball with parity breaking, we must introduce the axial/ chiral chemical potential [10]. At finite temperature, the transitions between

\footnotetext{
^e-mail: a.andrianov@spbu.ru

$\star \star$ e-mail: v.andrianov@spbu.ru

$\star \star \star$ e-mail: espriu@icc.ub.edu
} 
the vacuum states with different topological Chern-Simons numbers can be induced by a classical thermal activation process, so-called "sphaleron" [11]. In QCD matter, sphalerons are abundant [12] and induce the quark chirality non-conservation. There are some experimental indications of an abnormal dilepton excess in the range of low invariant masses and rapidities and moderate values of the transverse momenta (see the review in [13]), which can be thought of as a result of LPB in the medium (the details can be found in [14]). In particular, in heavy-ion collisions at high energies, with raising temperatures and baryon densities, metastable state can appear in the fireball with a nontrivial topological/axial charge $T_{5}$, which is related to the gluon gauge field $G_{i}$ :

$$
T_{5}(t)=\frac{1}{8 \pi^{2}} \int_{\text {vol. }} d^{3} x \varepsilon_{j k l} \operatorname{Tr}\left(G^{j} \partial^{k} G^{l}-i \frac{2}{3} G^{j} G^{k} G^{l}\right), \quad j, k, l=1,2,3,
$$

where the integration is over the finite fireball volume. Its jump $\Delta T_{5}$ can be associated with the space-time integral of the gauge-invariant Chern-Pontryagin density:

$$
\Delta T_{5}=T_{5}\left(t_{f}\right)-T_{5}(0)=\frac{1}{16 \pi^{2}} \int_{0}^{t_{f}} d t \int_{\text {vol. }} d^{3} x \operatorname{Tr}\left(G^{\mu v} \widetilde{G}_{\mu v}\right)
$$

It is known that the divergence of isosinglet axial quark current $J_{5, \mu}=\bar{q} \gamma_{\mu} \gamma_{5} q$ is locally constrained via the relation of partial conservation of axial current (PCAC) affected by the gluon anomaly:

$$
\partial^{\mu} J_{5, \mu}-2 \widehat{\imath}_{q} \bar{q} \gamma_{5} q=\frac{N_{f}}{2 \pi^{2}} \operatorname{Tr}\left(G^{\mu v} \widetilde{G}_{\mu \nu}\right) .
$$

This relation allows to find the connection of a nonzero topological charge with a non-trivial quark axial charge $Q_{5}^{q}$. Namely, integrating (3) over a finite volume of fireball we come to the equality

$$
\begin{aligned}
& \frac{d}{d t}\left(Q_{5}^{q}-2 N_{f} T_{5}\right) \simeq 2 i \int_{\text {vol. }} d^{3} x \widehat{m}_{q} \bar{q} \gamma_{5} q, \\
& Q_{5}^{q}=\int_{\text {vol. }} d^{3} x q^{\dagger} \gamma_{5} q=\left\langle N_{L}-N_{R}\right\rangle,
\end{aligned}
$$

where $\left\langle N_{L}-N_{R}\right\rangle$ stands for the vacuum averaged difference between left and right chiral densities of baryon number (chiral imbalance). Therefrom it follows that in the chiral limit and for a finite fireball volume the axial quark current is conserved in the presence of non-zero topological charge. If for the lifetime of fireball and the size of hadron fireball of order $L=5-10 \mathrm{fm}$, the created topological charge is non-zero, $\left\langle\Delta T_{5}\right\rangle \neq 0$, then it may be associated with a topological chemical potential $\mu_{\theta}$ or an axial chemical potential $\mu_{5}$ [10]. Thus we have

$$
\left\langle\Delta T_{5}\right\rangle \simeq \frac{1}{2 N_{f}}\left\langle Q_{5}^{q}\right\rangle \Longleftrightarrow \mu_{5} \simeq \frac{1}{2 N_{f}} \mu_{\theta} .
$$

Thus adding to the QCD Lagrangian the term $\Delta \mathcal{L}_{\text {top }}=\mu_{\theta} \Delta T_{5}$ or $\Delta \mathcal{L}_{q}=\mu_{5} Q_{5}^{q}$, we get the possibility of accounting for non-trivial topological fluctuations ("fluctons") in the nuclear (quark) fireball.

\section{Effective meson theories with LPB}

For the detection of LPB in the hadron fireball let us consider an effective theory describing the electromagnetic interactions in a fireball. In this case, we keep in mind mechanisms of hadron interactions which rely on vector dominance model [15]. 
The quark-meson interaction is described by the Lagrangian,

$$
\mathcal{L}_{\text {int }}=\bar{q} \gamma_{\mu} V^{\mu} q ; \quad V_{\mu} \equiv-e A_{\mu} Q+\frac{1}{2} g_{\omega} \omega_{\mu} \mathbf{I}_{q}+\frac{1}{2} g_{\rho} \rho_{\mu}^{0} \lambda_{3}+\frac{1}{\sqrt{2}} g_{\phi} \phi_{\mu} \mathbf{I}_{s}
$$

while $Q=\frac{\lambda_{3}}{2}+\frac{1}{6} \mathbf{I}_{q}-\frac{1}{3} \mathbf{I}_{s}, g_{\omega} \simeq g_{\rho} \equiv g \simeq 6<g_{\phi} \simeq 7.8$ and the values of the constants are extracted from the decays of the vector mesons. Here, $\mathbf{I}_{q}$ and $\mathbf{I}_{s}$ are the unit matrices in the non-strange and strange quark sectors, and $\lambda_{3}$ is a corresponding Gell-Mann matrix. The parity-odd contribution is given by the Chern-Simons term,

$$
\mathcal{L}_{\mathrm{CS}}(k)=-\frac{1}{4} \varepsilon^{\mu \nu \rho \sigma} \operatorname{Tr}\left[\hat{\zeta}_{\mu} V_{\nu}(x) V_{\rho \sigma}(x)\right]=\frac{1}{2} \operatorname{Tr}\left[\hat{\zeta} \epsilon_{j k l} V_{j} \partial_{k} V_{l}\right]
$$

which describes the mixing of photons and vector mesons under the local spatial parity breaking. We can obtain the relation $\zeta=N_{c} g^{2} \mu_{5} / 8 \pi^{2}$ where $N_{c}$ is a number of colors, and numerically $\zeta \simeq$ $1.5 \mu_{5}$.

The analysis of the massive Chern-Simons electrodynamics [10] has shown that in the case of an isosinglet pseudoscalar background field the spectrum of massive vector mesons splits into three polarizations with the masses $m_{V,+}^{2}<m_{V, L}^{2}<m_{V,-}^{2}$. The position of resonance poles for transverse polarizations of $\rho^{0}, \omega$ - mesons is shifted with the wave vector $|\vec{k}|$ and also a resonance broadening occurs that leads to an increased contribution of the dilepton production compared with the case of resonances in vacuum (for details, see [16]). Then the question arises. Could the splitting be measured in experiments with heavy-ion collisions?

For this purpose there should be analyzed an effect of anomalous dilepton pair production in the range of low invariant masses and rapidities and moderate transverse momenta which was established in a series of experiments with heavy-ion collisions in recent years [13]. It is well known that the angular distribution of leptons carries the information on polarizations. However, the current angular distribution studies based on full angular average do not seem to detect possible parity-odd effects.

In order to isolate the transverse polarizations, we perform different cuts choosing the angle $\theta_{A}$ for the analysis (see figure 1) and study the variations of the $\rho$ (and $\omega$ ) spectral functions. A quite visible
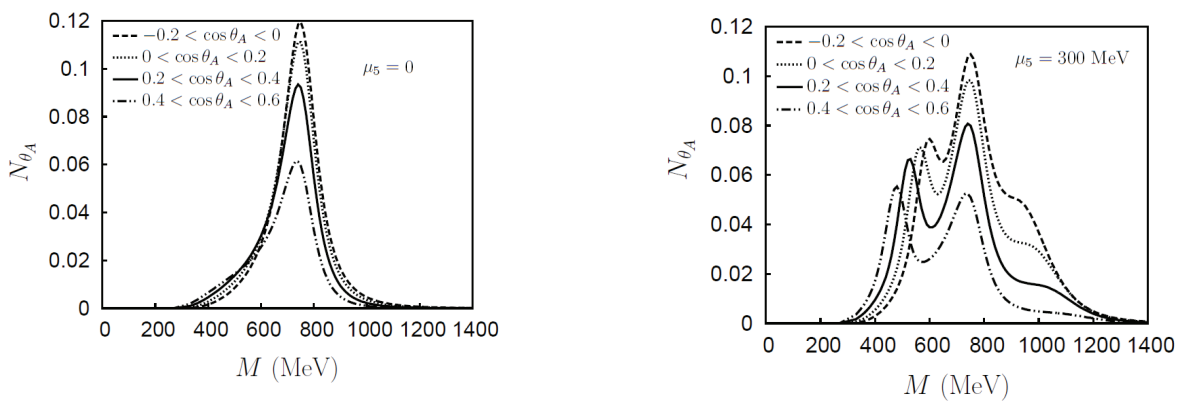

Figure 1. $\rho$ spectral function depending on the dielectron invariant mass $M$ in vacuum $\left(\mu_{5}=0\right)$ and in a paritybreaking medium with $\mu_{5}=300 \mathrm{MeV}$ for different ranges of the angle $\theta_{A}$ between the two outgoing leptons in the laboratory frame [16].

secondary peak appears in a $P$-odd medium! To isolate the transverse polarizations in the spectrum, 
we selected different angle sectors and studied the changes in the $\rho$-meson spectral function. Various experimental possibilities for its identification were discussed in [16].

Thus a signal (a phase) with spatial parity breaking in heavy-ion collisions (in a fireball) can be sought in experiments "event by event" using an excess yield of dilepton pairs and predominantly with different circular polarizations outside the resonance region of the $\rho$ - and $\omega$-meson invariant masses.

\section{Effective scalar meson Lagrangian with axial chemical potential}

In the environment with chiral chemical potential the scalar sector can be described by using the spurion technique in the chiral Lagrangian with an isosinglet $\mu_{5}$ [17],

$$
D_{v} \Longrightarrow D_{v}-i\left\{\mathbf{I}_{q} \mu_{5} \delta_{0 v}, \cdot\right\}=D_{v}-2 i \mathbf{I}_{q} \mu_{5} \delta_{0 v},
$$

where for three light flavors $\mathbf{I}_{q} \equiv \operatorname{diag}[1,1,0]$ in accordance with the preceding discussion. An effective Lagrangian is required to include the lightest isoscalar degrees of freedom such as $\sigma$ and $a_{0}(980)$, which will be mixed with their pseudoscalar partners $\eta, \eta^{\prime}$ and $\pi$, respectively.

In the lowest order of chiral expansion such an effective Lagrangian reads,

$$
\begin{gathered}
\mathcal{L}=\frac{1}{4} \operatorname{Tr}\left(D_{\mu} H D^{\mu} H^{\dagger}\right)+\frac{B}{2} \operatorname{Tr}\left[\hat{m}\left(H+H^{\dagger}\right)\right]+\frac{M^{2}}{2} \operatorname{Tr}\left(H H^{\dagger}\right) \\
-\frac{\lambda_{1}}{2} \operatorname{Tr}\left[\left(H H^{\dagger}\right)^{2}\right]-\frac{\lambda_{2}}{4}\left[\operatorname{Tr}\left(H H^{\dagger}\right)\right]^{2}+\frac{c}{2}\left(\operatorname{det} H+\operatorname{det} H^{\dagger}\right) \\
+\frac{d_{1}}{2} \operatorname{Tr}\left[\hat{m}\left(H H^{\dagger} H+H^{\dagger} H H^{\dagger}\right)\right]+\frac{d_{2}}{2} \operatorname{Tr}\left[\hat{m}\left(H+H^{\dagger}\right)\right] \operatorname{Tr}\left(H H^{\dagger}\right)
\end{gathered}
$$

where the $S U(3)_{L} \times S U(3)_{R}$ chirally invariant part is parameterized by constants $M, \lambda_{1,2}, c$ and soft chiral symmetry breaking is realized with the help of vertices containing $\hat{m}=\operatorname{diag}\left[m_{u}, m_{d}, m_{s}\right]$ and constants $B, d_{1,2}$. The convenient splitting of scalar fields is given by

$$
H=\xi \Sigma \xi, \quad \xi=\exp \left(i \frac{\Phi}{2 f}\right), \quad \Phi=\lambda^{a} \phi^{a}, \quad \Sigma=\lambda^{b} \sigma^{b}
$$

The v.e.v. of the neutral scalars are defined as $v_{i}=\left\langle\Sigma_{i i}\right\rangle$ where $i=u, d$, s, and, in the chiral limit $\hat{m}=0$, satisfy the following gap equations:

$$
\begin{gathered}
\left(2 \mu_{5}^{2}+M^{2}\right)-2\left(\lambda_{1}+\lambda_{2}\right) v_{q}^{2}-\lambda_{2} v_{s}^{2}+c v_{s}=0 \\
\left(-2 \lambda_{2}+\frac{c}{v_{s}}\right) v_{q}^{2}+M^{2}-\left(2 \lambda_{1}+\lambda_{2}\right) v_{s}^{2}=0
\end{gathered}
$$

where for non-vanishing isosinglet $\mu_{5}$ we find our solutions to be $v_{u}=v_{d} \equiv v_{q} \neq v_{s}$.

Evidently for large $\mu_{5}$ the correct solution has the following asymptotics,

$$
v_{q}^{2}=\frac{2 \mu_{5}^{2}+M^{2}+\frac{c^{2}}{4 \lambda^{2}}}{2\left(\lambda_{1}+\lambda_{2}\right)}+O\left(1 / \mu_{5}^{2}\right), \quad v_{s}=\frac{c}{2 \lambda_{2}}+O\left(1 / \mu_{5}^{2}\right) .
$$

These solutions are depicted in the figure 2.

We stress that the consistent solution with a finite limit for strange quark constituent mass $v_{s} \ll v_{q}$ exists if the term breaking $U(1)_{A}$ symmetry is in action. Meantime the constituent masses for light $u, d$ flavours are growing with chiral chemical potential, first as $\sim \mu_{5}^{2}$ and next as $\sim \mu_{5}$. Moreover even if the spontaneous chiral symmetry breaking is absent for $M^{2}<0$ in parity-even environment this CSB will be triggered by chiral imbalance.

The lattice computations perfectly confirm [18] this behavior against chiral chemical potential. 


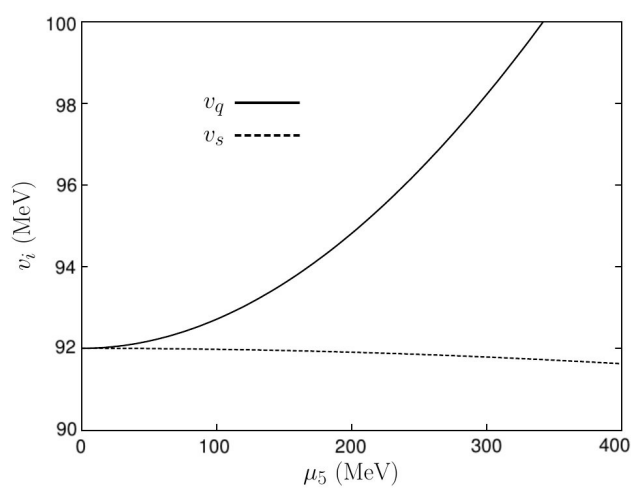

Figure 2. Vacuum condensates in the presence of chiral imbalance [17].

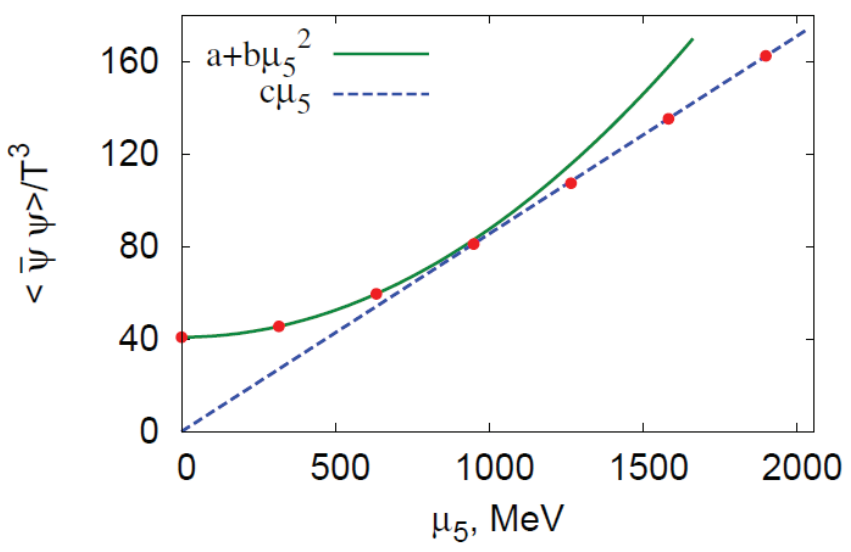

Figure 3. Chiral condensate in two-color QCD lattice (red dots) as a function of $\mu_{5}$ in the confinement phase (T $=158 \mathrm{MeV}$ ) [18].

For further purposes we need the non-strange meson sector and $\eta_{s}$

$$
\begin{gathered}
\Phi=\left(\begin{array}{ccc}
\eta_{q}+\pi^{0} & \sqrt{2} \pi^{+} & 0 \\
\sqrt{2} \pi^{-} & \eta_{q}-\pi^{0} & 0 \\
0 & 0 & \sqrt{2} \eta_{s}
\end{array}\right), \Sigma=\left(\begin{array}{ccc}
v_{u}+\sigma+a_{0}^{0} & \sqrt{2} a_{0}^{+} & 0 \\
\sqrt{2} a_{0}^{-} & v_{d}+\sigma-a_{0}^{0} & 0 \\
0 & 0 & v_{s}
\end{array}\right) \\
\left(\begin{array}{l}
\eta_{q} \\
\eta_{s}
\end{array}\right)=\left(\begin{array}{cc}
\cos \psi & \sin \psi \\
-\sin \psi & \cos \psi
\end{array}\right)\left(\begin{array}{c}
\eta \\
\eta^{\prime}
\end{array}\right) .
\end{gathered}
$$

For $\mu_{5}=0$, in the chiral limit $\hat{m}=0$ we adopt $v_{u}=v_{d}=v_{s}=v_{0} \equiv f_{\pi} \approx 92 \mathrm{MeV}$ and the notations $m \equiv m_{q}=\left(m_{u}+m_{d}\right) / 2$ and $m / m_{s} \simeq 1 / 25$. . The coupling constants (in units of MeV) were fitted to phenomenology assuming isospin symmetry with the help of $\chi^{2}$ minimization (MINUIT).

One, of course, should not expect the range of applicability of dim-4 meson Lagrangian for chemical potentials larger than few hundreds of $\mathrm{MeV}$. Nevertheless the predictions for lightest states, pions can be well controlled within the chiral perturbation theory. 
Let us now consider the QCD-inspired scalar meson model with axial chemical potential [17] for a description of some properties of low-energy mesons in medium and how it manifests itself in LPB. First of all the axial chemical potential is introduced and treated as a constant time component of an isosinglet axial-vector field in the non-strange sector. For lightest isotriplet pseudoscalar $\pi$ and scalar $a_{0}$ states the piece of the effective Lagrangian that is bilinear in fields looks as follows

$$
\mathcal{L}=\frac{1}{2}\left(\partial a_{0}\right)^{2}+\frac{1}{2}(\partial \pi)^{2}-\frac{1}{2} m_{1}^{2} a_{0}^{2}-\frac{1}{2} m_{2}^{2} \pi^{2}-4 \mu_{5} a_{0} \dot{\pi}
$$

Evidently new eigenstates $\tilde{\pi}$ arise from the mixture of scalars and pseudoscalars then for big 3momentum $\tilde{\pi}$ become massless and further on tachyonic [17]. For the same 3-momentum the quasiscalar state $\sigma$ looks stable.

\section{Chemical potentials in the NJL model}

In [19] we incorporated both a vector and an axial chemical potentials in the Nambu-Jona-Lasinio (NJL) model with the purpose of unraveling the landscape of different stable phases of the theory. It turns out that the inclusion of $\mu_{5}$ changes radically the phase structure of the model and shows that $\mu$ is not a key player in ushering a thermodynamically stable phase where parity is violated in the NJL model, but $\mu_{5}$ is. It leads to a non-trivial dependence of the scalar condensate in the chirally broken phase (see figure 4).
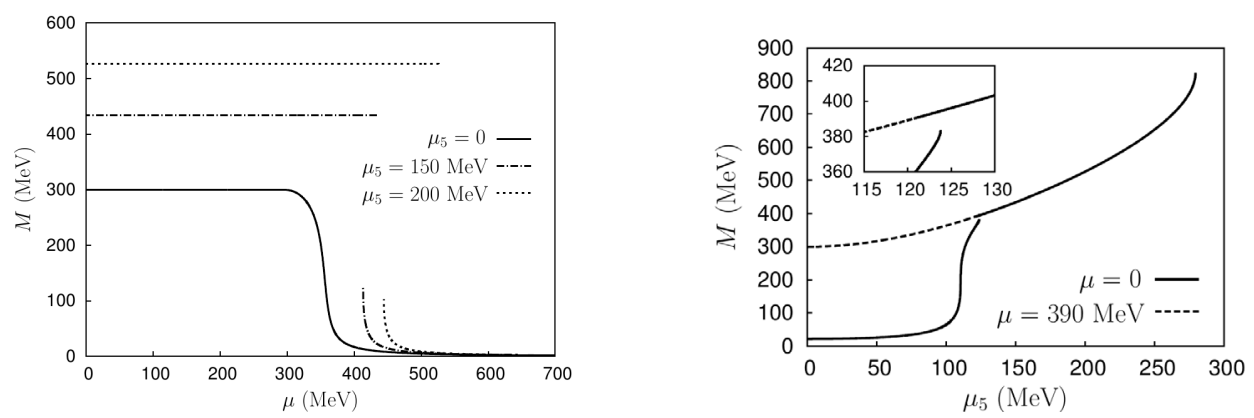

Figure 4. Evolution of the constituent quark mass $M$ [19]: left panel- depending on $\mu$ for different values of the axial chemical potential $\mu_{5}$; right panel- depending on $\mu_{5}$ for different values of the chemical potential $\mu$.

Critical temperature with axial potential switched on was computed on lattice [18] and a fairly well correspondence with the results of [19] was established. The main result is that with increasing chiral chemical potential the dynamical mass and critical temperature raise up.

\section{Conclusions and outlook}

In this talk we described a possibility of local spatial parity breaking emerging in a dense hot baryon medium (fireball) in heavy-ion collisions at high energies. We stress that LPB is not forbidden by any physical principle in QCD at finite temperature/density. We suggested a generalized Lagrangian of the vector meson dominance model in the presence of the Chern-Simons interaction. It turns out that the spectrum of massive vector mesons splits into three components with different polarizations and with different effective masses $m_{V,+}^{2}<m_{V, L}^{2}<m_{V,-}^{2}$ and a resonance broadening occurs that leads to 
an increase of the spectral contribution to the dilepton production as compared with the vacuum state. The proposed mechanism for generating local spatial parity breaking helps to explain qualitatively and quantitatively the anomalous yield of dilepton pairs in the CERES, PHENIX, STAR, NA60, and ALICE experiments.

Besides, LPB enhances dynamical chiral symmetry breaking in QCD: chiral condensates, critical temperature of chiral symmetry restoration are increasing with chiral chemical potential. Accordingly, LPB modifies dispersion laws for scalar and vector mesons: lightest "pseudoscalar" mesons tend to massless states in flight, vector meson polarizations split with different in-flight masses.

\section{Acknowledgements}

A.A. expresses his appreciation to the organizing Committee of the XXIII International Baldin Seminar "Relativistic Nuclear Physics and Quantum Chromodynamics", for the hospitality and organization of this conference. This work has been partially supported through grants FPA2013-46570, 2014-SGR-104 and Consolider CPAN. Funding was also provided by the Spanish MINECO under project MDM-2014-0369 of ICCUB (Unidad de Excelencia 'Maria de Maeztu'). A.A. and V.A. were supported by Grant RFBR project 16-02-00348 and also got a financial support of SPbSU, in particular, by projects 11.42.1049.2016, 11.42.1051.2016.

\section{References}

[1] P. Jacobs, D. Kharzeev, B. Muller, J. Nagle, K. Rajagopal and S. Vigdor, [arXiv:0705.1930 [nucl-ex]]

[2] J.-P. Blaizot, F. Gelis, J. -F. Liao, L. McLerran and R. Venugopalan, Nucl. Phys. A, 873, 68 (2012)

[3] A. Andronic et al., Nucl. Phys. A, 837, 65 (2010)

[4] B. Abelev, et al., Phys. Rev. C, 81, 054908 (2010)

[5] B. Abelev et al. (ALICE Collaboration), Phys. Rev. Lett. 110, 012301 (2013)

[6] D. Kharzeev, Phys. Lett. B 633, 260 (2006)

[7] D. E. Kharzeev, L. D. McLerran, and H. J. Warringa, Nucl. Phys. A 803, 227 (2008)

[8] K. Fukushima, D. E. Kharzeev, and H. J. Warringa, Phys. Rev. D 78, 074033 (2008)

[9] D. E. Kharzeev, Prog. Part. Nucl. Phys. 75, 133 (2014)

[10] A. A. Andrianov, V. A. Andrianov, D. Espriu and X. Planells, Phys. Lett. B 710, 230 (2012)

[11] F.R. Klinkhamer, N.S. Manton, Phys. Rev. D 30, 2212 (1984). V.A. Kuzmin, V.A. Rubakov, M.E. Shaposhnikov, Phys. Lett. B 155, 36 (1985)

[12] L. D. McLerran, E. Mottola and M. E. Shaposhnikov, Phys. Rev. D 43, 2027 (1991)

[13] I. Tserruya, Landolt-Börnstein 23, 176 (2010)

[14] A. A. Andrianov, V. A. Andrianov, D. Espriu, X. Planells, Theor. Math. Phys., 170, 17 (2012). A. A. Andrianov, V. A. Andrianov, Theor. Math. Phys. 185, 1370 (2015)

[15] J. J. Sakurai, Ann. Phys. 11, 1 (1960)

[16] A. A. Andrianov, V. A. Andrianov, D. Espriu, and X. Planells, Phys. Rev. D 90, 034024 (2014)

[17] A. A. Andrianov, D. Espriu \& X. Planells, Eur. Phys. J. C 73, 2294 (2013)

[18] V. V. Braguta, V. A. Goy, E. M. Ilgenfritz, A. Yu. Kotov, A. V. Molochkov, M. Muller-Preussker, and B. Petersson. JHEP, 06, 094 (2015). V.V. Braguta et al. Phys. Rev. D 93, 034509 (2016). V.V. Braguta et al. AIP Conf. Proc., 1701, 060002 (2016). V.V. Braguta and A.Y. Kotov, Phys. Rev. D 93, 105025 (2016)

[19] A. A. Andrianov, D. Espriu, and X. Planells, Eur. Phys. J. C 74, 2776 (2014) 